\title{
AUTOMATIC COMPARISON OF CHILDREN'S AND ADULT SONGS SUPPORTS THE VOCAL MISTUNING THEORY OF SCALE ORIGINS
}

\author{
Shoichiro Sato, Shinya Fujii, Patrick E. Savage \\ Keio University, Shonan Fujisawa Campus \\ \{s14411ss, sfujii, psavage\}@sfc.keio.ac.jp
}

\begin{abstract}
Human music uses diverse scales, but there are some commonalities shared throughout much of the world's scales. The "vocal mistuning" hypothesis proposes that cross-cultural regularities in musical scales arise from imprecision in vocal tuning. In order to test this hypothesis, we conducted automatic comparative analysis of 70 matched children's and adult songs from 35 areas around the world. We found that children's songs tend to have fewer scale degrees than adults and narrower melodic ranges than adult songs, consistent with motor limitations due to their earlier developmental stage. These results suggest that some universal aspects of musical scales may be caused by motor constraints rather than evolutionary adaptations for music perception.
\end{abstract}

\section{INTRODUCTION}

Human songs throughout the world use diverse scales, but are there musical laws or biological constraints that underlie this diversity? Theories of the origin of scales have been based on perceptual theories involving integer ratios since the time of Pythagoras [1]. However, these theories are generally based on tunable instruments, and some are skeptical as to whether this theory applies to vocal song, recognized as the most ancient and universal instrument of human music [3]. One alternative theory is that scales arise not from perceptual constraints regarding integer ratios but instead due to production constraints on how precisely the voice can generate pitches [3]. This can be seen as a special case of the "motor constraint hypothesis", which proposes that many musical universals are not evolutionary adaptations for human music but simply byproducts of the way music is produced $[6,8]$. The vocal mistuning theory argues that the universal tendency to use sparse scales with 7 or fewer scale degrees [4] is because singing is too imprecise to allow accurate production of scales using more than 7 scale degrees. It predicts a negative correlation between tuning precision and number of scale degrees.

In order to test the "vocal mistuning" theory of scales, here we use an automatic method to compare global children songs and adult songs from the perspective of scales and melodic range. Since children's vocal-motor control is still developing, children should have less precise pitch than adult songs, and thus the vocal mistuning theory predicts that their scales should have fewer scales degrees. Moreover, a more general motor constraint theory predicts that children's songs should have a smaller melodic range than adult songs due to their more limited vocal range. In previous research, scales, melodic ranges, and other aspects were analyzed from 100 children's songs from around the world [2], but this study was limited to manual analysis and children's songs were not directly compared with adult songs. In this study, we use automatic analysis to directly compare children's and adult songs using a matched global sample.

\section{METHODS}

\subsection{Song sample}

For this preliminary analysis, the same 35 songs used in [6], collected from Garland Encyclopedia of World Music, are analyzed as adult vocal songs. 35 children songs from the same geographic areas were selected from Le chant des enfants $d u$ monde [5]. All recordings are monophonic or unison, excluding songs with instrumental accompaniment and polyphonic singing (because automatic pitch estimation cannot yet be performed for polyphonic music).

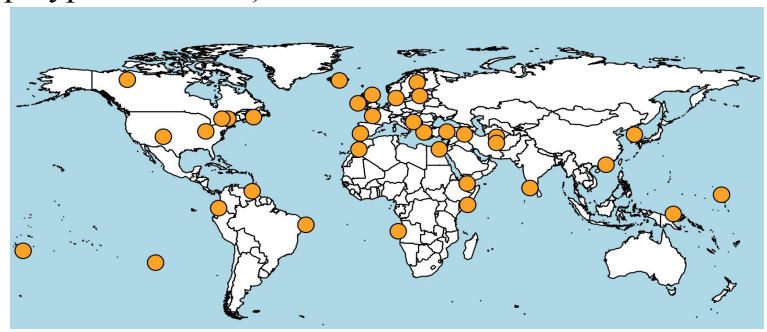

Figure 1. Map of the 35 areas from which the 70 matched adult and children's songs originated.

\subsection{Scale analysis}

We used Tarsos [7] to automatically identify the number of scale degrees because it was designed explicitly for automatic analysis of any scales from around the world without imposing any culture-specific theories. Tarsos first extracts the pitch histogram, then collapses this pitch histogram across octaves to create a pitch class histogram, expressed in cents [2] ranging from 0-1200, and finally automatically picks peaks (see Fig. 2). We used Tarsos's default YIN pitch estimation algorithm. 
Peak picking was based on the default values for window and threshold with some manual adjustment (in the future we plan to evaluate optimal window and threshold values to allow objective peak picking without the need for manual adjustment).

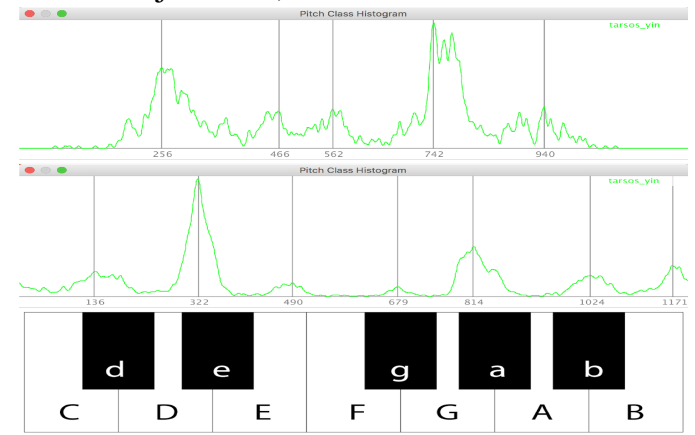

Figure 2. Automatic analysis of traditional West African scales: "Sigereti Fe Bara" (top, children's song, five scale degrees) and "Vai Call to Prayer" (bottom, adult song, seven scale degrees). Vertical lines represent automatically detected scale degrees.

\subsection{Melodic range analysis}

The melodic range was estimated by the visualization of melograph representation (Fig. 3). Each estimated pitch is plotted over time in absolute cents and visualized as a density of pitch distribution. Each song's melodic range is obtained by calculating approximate differences between the highest and the lowest pitches and performed t-test. Pitch estimation errors due to background noises and harmonic overtones are ignored (currently manually; in the future we plan to automate this).

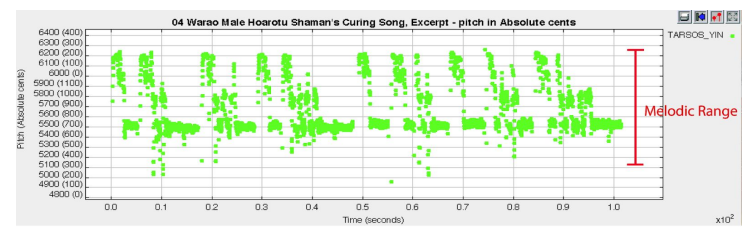

Figure 3. An example of melodic range analysis for the "Warao Male Hoarotu Shaman's Curing Song" from Venezuela.

\section{PRELIMINARY RESULTS}

Thorough analyzing 35 adult and 35 children's songs from around the world, the number of scale degrees and melodic range are compared with each other. Figure 4 shows the number of scale degrees separately for children's and adult songs. Children's songs tended to have fewer scales degrees than adult songs.

Furthermore, the mean absolute melodic ranges of children's songs was 953 cents (i.e., more than a minor 7th) while that of adult's song was 1353 cents (i.e., more than a minor 9th). The difference between the two groups was significant with adult songs exhibiting a melodic range over the $40 \%$ greater than children's songs $(\mathrm{p}<0.01)$.

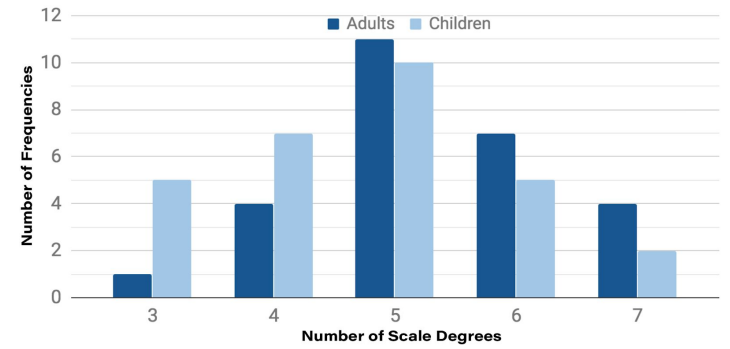

Figure 4. The comparison of number of scale degrees between children's and adult songs.

\section{DISCUSSION/FUTURE WORK}

Through this comparative analysis, children's songs show relatively smaller melodic ranges and relatively sparser scales than adult songs for a balanced global sample. These results support the predictions of the vocal mistuning and motor constraint hypotheses [3, 8]. However, to more directly test the predictions of the vocal mistuning hypothesis for scale origins against the competing integer ratio hypothesis [1], more detailed analysis of scale tunings and precise measurements of tuning imprecision will be required, using larger samples.

\section{ACKNOWLEDGMENTS}

This work was funded by a Keio Research Institute at SFC Startup Grant and a Keio Gijuku Academic Development Fund Individual Grant to P.E.S.

\section{REFERENCES}

[1] D. L. Bowling and D. Purves, "A Biological Rationale for Musical Consonance," Proc. Natl. Acad. Sci. U.S.A., vol. 112, no. 36, pp. 11155-11160, 2015.

[2] S. J. Pai, "Discovering Musical Characteristic Of Children's song From Various Parts of The World" MA thesis, University of British Columbia, 2005

[3] P. Q. Pfordresher \& S. Brown, "Vocal mistuning reveals the origin of musical scales." Journal of Cognitive Psychology, vol. 29, no. 1, pp. 35-52, 2017.

[4] P. E. Savage, S. Brown, E. Sakai, and T. E. Currie, "Statistical Universals Reveal the Structures and Functions of Human Music," Proc. Natl. Acad. Sci. U. S. A., vol. 112, no. 29, pp. 8987-8992, 2015.

[5] F. Corpataux, "Le Chant des enfants du monde" [11 CDs], ARION, 1993-2006

[6] P. E. Savage, A. T. Tierney, \& A. D. Pate, "Global Music Recordings Support the Motor Constraint Hypothesis for Human and Avian Song Contour" Music Perception: An Interdisciplinary Journal, 34(3), 327-334, 2017

[7] J. Six, O. Cornelis, \& M. Leman, "Tarsos, a modular platform for precise pitch analysis of Western and non-Western music" Journal of New Music Research, 42:2, 113-129, 2013

[8] A. T. Tierney, F. A. Russo \& A. D. Pate, "The motor origins of human and avian song structure." Proceedings of the National Academy of Sciences, 2011 